\title{
APPLICATION OF CUBIC BOX SPLINE WAVELETS IN THE ANALYSIS OF SIGNAL SINGULARITIES
}

\author{
WALDEMAR RAKOWSKI ${ }^{a}$ \\ ${ }^{a}$ Faculty of Management \\ Białystok University of Technology, ul. Stefana Tarasiuka 2, 16-001 Kleosin, Poland \\ e-mail: w.rakowski@pb.edu.pl
}

\begin{abstract}
In the subject literature, wavelets such as the Mexican hat (the second derivative of a Gaussian) or the quadratic box spline are commonly used for the task of singularity detection. The disadvantage of the Mexican hat, however, is its unlimited support; the disadvantage of the quadratic box spline is a phase shift introduced by the wavelet, making it difficult to locate singular points. The paper deals with the construction and properties of wavelets in the form of cubic box splines which have compact and short support and which do not introduce a phase shift. The digital filters associated with cubic box wavelets that are applied in implementing the discrete dyadic wavelet transform are defined. The filters and the algorithme à trous of the discrete dyadic wavelet transform are used in detecting signal singularities and in calculating the measures of signal singularities in the form of a Lipschitz exponent. The article presents examples illustrating the use of cubic box spline wavelets in the analysis of signal singularities.
\end{abstract}

Keywords: cubic box splines, wavelets, dyadic wavelet transform, singularity detection.

\section{Introduction}

An important issue in signal processing is to determine a representation of the signal, which simplifies further extraction of information contained in that signal. Often, the relevant characteristics of the signal arise from the existence of points where the signal varies rapidly in value. The derivative of the signal may be useful in determining the location of these points. However, numerical differentiation of a discrete-time signal, especially a noisy one, is an ill-conditioned problem. The initial smoothing of the signal by calculating the integral,

$$
\int_{-\infty}^{+\infty} f(u) \theta(u-t) \mathrm{d} u=f \star \bar{\theta}(t)
$$

may be helpful in solving the problem where $f(t)$ is the signal processed, $\theta(t)$ is the smoothing function with a short support in relation to the domain of the signal or rapidly vanishing as a Gaussian function, and where

$$
\begin{aligned}
\int_{-\infty}^{+\infty} \theta(t) \mathrm{d} t & \neq 0, \\
\bar{\theta}(t) & =\theta(-t),
\end{aligned}
$$

$$
f \star \bar{\theta}(t)=\int_{-\infty}^{+\infty} f(u) \bar{\theta}(t-u) \mathrm{d} u
$$

The derivative of the smoothed signal is equal to a convolution of the signal with the derivative of the smoothing function (see Section 2),

$$
\frac{\mathrm{d}}{\mathrm{d} t}[f \star \bar{\theta}(t)]=f \star \bar{\theta}^{\prime}(t),
$$

where $\bar{\theta}^{\prime}(t)=\mathrm{d} \bar{\theta}(t) / \mathrm{d} t$. It may be useful to smooth the signal using the scaled smoothing function and to differentiate the smoothed signal in different scales,

$$
\frac{\mathrm{d}}{\mathrm{d} t} \int_{-\infty}^{+\infty} f(u) \frac{1}{\sqrt{s}} \theta\left(\frac{u-t}{s}\right) \mathrm{d} u=\frac{\mathrm{d}}{\mathrm{d} t}\left[f \star \bar{\theta}_{s}(t)\right],
$$

where $s>0$ is the scale factor. Initial signal smoothing and differentiation of the smoothed signal are particularly useful in locating places where the signal has a sharp transition. Such places are called singular points, and their determination is referred to as signal singularity detection (formal definition in Section 6), which applies to one-dimensional signals as well as two-dimensional ones (images). 
As far as the images are concerned, edges are a good illustration of singular places. Smoothing introduces inflection points in the signal where the first derivative has local extremes and the second one has zero-crossings. Babaud et al. (1986) show that the roots of the second derivative of the smoothed signal, i.e., the points $(t, s)$ in the half-plane $0 t s(t \in \mathbb{R}, s>0)$ that satisfy the equation

$$
\frac{\mathrm{d}^{2}}{\mathrm{~d} t^{2}}\left[f \star \bar{\theta}_{s}(t)\right]=0,
$$

when the smoothing function $\theta(t)$ is a Gaussian function, form outlines that end at the singular points of the signal as the scale decreases to zero, $s \rightarrow 0$. Points on a coarse scale that satisfy Eqn. (4) allow us to identify (suggest the existence of) singular positions, while points on a fine scale can localize, i.e., determine, the value of the variable $t$ where the singularity occurs. The ability to identify singularities in a coarse scale is important because the noise present in the signal may suggest the existence of additional points corresponding to the contours that appear in fine scales. At coarse scales, the noise is filtered and the appearing contours correspond to real singular points of the signal. However, at a coarse scale, due to strong signal smoothing, it may be difficult to identify the points of local extremes, particularly in the discrete form, as the smoothed signal may have the same value at several neighboring points. In such a case, identifying the points forming the contours ending in singular points is allowed by extremes in fine scales.

Smoothing and differentiation of the smoothed signal are directly related to the calculation of the wavelet transform of a signal using the wavelet equal to the derivative of the smoothing function. The mathematical foundation of singularity detection using the wavelet transform was presented in several papers. Namely, Mallat (1991) shows the application of the wavelet called the Mexican hat (the second derivative of a Gaussian) for singularity detection. Mallat and Hwang (1992) use a quadratic box spline wavelet. Mallat and Zhong (1992) define filter coefficients corresponding to the quadratic box spline wavelet.

In this paper, we present the design and application of digital filters corresponding to the scaling function and the wavelet as a cubic box spline to detect signal singularities. Box splines have a compact and short support; the corresponding filters have finite and short impulse responses. The cubic box spline is an even function, and as a consequence there is no phase shift introduced by the filter corresponding to that wavelet.

1.1. Definitions. $\mathbb{Z}$ denotes the set of integers, $L^{2}(\mathbb{R})$ means the space of measurable, square-integrable one-dimensional functions; $l^{2}(\mathbb{Z})$ means the space of square-summable sequences, also known as the space of finite energy sequences.
The convolution of two continuous-time signals $f(t) \in L^{2}(\mathbb{R})$ and $g(t) \in L^{2}(\mathbb{R})$ has the following symbol and definition:

$$
f \star g(t)=\int_{-\infty}^{+\infty} f(u) g(t-u) \mathrm{d} u .
$$

The convolution of two discrete-time signals $f[n] \in l^{2}(\mathbb{Z})$ and $g[n] \in l^{2}(\mathbb{Z})$ has the following symbol and definition:

$$
f \star g[n]=\sum_{k=-\infty}^{+\infty} f[k] g[n-k] .
$$

The reversed signal in relation to the continuous-time signal $f(t)$ is

$$
\bar{f}(t)=f(-t) .
$$

The reversed signal in relation to the discrete-time signal $f[n]$ is

$$
\bar{f}[n]=f[-n] .
$$

The Fourier transform of a continuous-time signal $f(t)$ is defined as

$$
F(j \omega)=\int_{-\infty}^{+\infty} f(t) e^{-j \omega t} \mathrm{~d} t .
$$

The Fourier transform of a discrete-time signal $f[n]$ is defined as

$$
F\left(e^{j \omega}\right)=\sum_{n=-\infty}^{+\infty} f[n] e^{-j \omega n}
$$

The scaled function $f_{s}(t)$ is defined as

$$
f_{s}(t)=\frac{1}{\sqrt{s}} f\left(\frac{t}{s}\right) .
$$

The scaled and translated function $f_{u, s}(t)$ is defined as

$$
f_{u, s}(t)=\frac{1}{\sqrt{s}} f\left(\frac{t-u}{s}\right) \text {. }
$$

$\theta^{\prime}(t)$ represents the first derivative of the function $\theta(t)$, thus $\theta^{\prime}(t)=\mathrm{d} \theta(t) / \mathrm{d} t ; \theta^{\prime \prime}(t)$ represents the second derivative of the function $\theta(t)$, thus $\theta^{\prime \prime}(t)=\mathrm{d}^{2} \theta(t) / \mathrm{d} t^{2}$.

\section{Wavelet analysis}

In a wavelet analysis, there are two functions: a scaling function $\phi(t)$ and a wavelet $\psi(t)$, and two digital filters: a low-pass filter $h$ and a high-pass filter $g$. In terms of signal processing, the scaling function is an impulse response of a low-pass filter and the wavelet is an impulse response of a band-pass filter. Both the wavelet and the scaling function are characterized by fast decay, e.g., an exponential or compact support. Relations between the 
scaling function, the wavelet and the filters are described by the following two equations:

$$
\begin{aligned}
& \frac{1}{\sqrt{2}} \phi(t / 2)=\sum_{n} h[n] \phi(t-n), \\
& \frac{1}{\sqrt{2}} \psi(t / 2)=\sum_{n} g[n] \phi(t-n) .
\end{aligned}
$$

Equation (13) is called the dilation equation. A characteristic feature of the scaling function is a non-zero value of the following integral:

$$
\int_{-\infty}^{+\infty} \phi(t) \mathrm{d} t \neq 0
$$

It is assumed that $\|\phi(t)\|=1$. The coefficient $1 / \sqrt{2}$ ensures preservation of the norm of the scaled function in the space $L^{2}(\mathbb{R})$. In the frequency domain, the scaling equation takes the form of

$$
\Phi(j 2 \omega)=\frac{1}{\sqrt{2}} H\left(e^{j \omega}\right) \Phi(j \omega) .
$$

The Fourier transform of the scaling function as a transfer function of a low-pass filter is not equal to zero for $\omega=0$, i.e., $\Phi(j 0) \neq 0$. It is assumed that $H\left(e^{j 0}\right)=\sqrt{2}$, which means that $\sum_{n=-\infty}^{+\infty} h[n]=\sqrt{2}$. The dilation equation allows calculating the low-pass filter $h$ for a given scaling function $\phi(t)$.

Equation (14) is called the wavelet equation. The main feature of the wavelet is a vanishing integral of the wavelet, i.e.,

$$
\int_{-\infty}^{+\infty} \psi(t) \mathrm{d} t=0
$$

In the frequency domain, the wavelet equation takes the form

$$
\Psi(j 2 \omega)=\frac{1}{\sqrt{2}} G\left(e^{j \omega}\right) \Phi(j \omega) .
$$

Since $\Phi(j 0) \neq 0$, the number of zeros $p$ of the transfer function $G(j \omega)$ for $\omega=0$ determines the number of zeros of the Fourier transform of $\Psi(j \omega)$ for $\omega=0$. It is equivalent to the fact that the wavelet has $p$ vanishing moments, thus

$$
\int_{-\infty}^{+\infty} t^{k} f(t) \mathrm{d} t=0, \quad 0 \leq k \leq p-1 .
$$

This property indicates that the wavelet is orthogonal to the polynomial of degree $k \leq p-1$. The wavelet equation allows calculating the wavelet for a given scaling function $\phi(t)$ and a given high-pass filter $g$.

A wavelet transform of a continuous-time signal (a continuous spatial variable) is a function of two variables and is a measure of the similarity between the signal $f(t)$ and the wavelet $\psi_{u, s}(t)$ at scale $s$, shifted to the time instant $t=u$. The formal definition of a wavelet transform of a continuous-time signal is

$$
\begin{aligned}
W f(u, s) & =\int_{-\infty}^{+\infty} f(t) \frac{1}{\sqrt{s}} \psi\left(\frac{t-u}{s}\right) \mathrm{d} t \\
& =\int_{-\infty}^{+\infty} f(t) \frac{1}{\sqrt{s}} \bar{\psi}\left(\frac{u-t}{s}\right) \mathrm{d} t
\end{aligned}
$$

and has the following symbolic notation:

$$
W f(u, s)=f \star \bar{\psi}_{s}(u) .
$$

The coefficient $1 / \sqrt{s}$ that appears before the scaled and shifted wavelet preserves the norm of the scaled wavelet in the space $L^{2}(\mathbb{R})$.

The relationship between the continuous-time wavelet transform and a derivative of the smoothed signal is shown by the following calculations:

$$
\begin{aligned}
\frac{\mathrm{d}}{\mathrm{d} t}\left[f \star \bar{\theta}_{s}(t)\right] & =\frac{\mathrm{d}}{\mathrm{d} t} \int_{-\infty}^{\infty} f(u) \frac{1}{\sqrt{s}} \bar{\theta}\left(\frac{t-u}{s}\right) \mathrm{d} u \\
& =\int_{-\infty}^{\infty} f(u) \frac{1}{\sqrt{s}}\left[\frac{d}{\mathrm{~d} t} \bar{\theta}\left(\frac{t-u}{s}\right)\right] \mathrm{d} u \\
& =\frac{1}{s} \int_{-\infty}^{\infty} f(u) \bar{\theta}_{s}^{\prime}(t-u) \mathrm{d} u \\
& =\frac{1}{s} f \star \bar{\theta}_{s}^{\prime}(t)
\end{aligned}
$$

The last equation means that the derivative of the signal smoothed by the scaled smoothing function is equal to the convolution of the signal with a derivative of the scaled smoothing function (with the accuracy to coefficient $1 / s$ ).

We define the wavelet in the form of a derivative of the smoothing function with a minus sign,

$$
\psi(t)=-\theta^{\prime}(t)
$$

which is equivalent to

$$
\begin{gathered}
\bar{\psi}(t)=\bar{\theta}^{\prime}(t), \\
\bar{\psi}_{s}(t)=\bar{\theta}_{s}^{\prime}(t) .
\end{gathered}
$$

Taking into account (22) and (25), we obtain

$$
\frac{\mathrm{d}}{\mathrm{d} t}\left[f \star \bar{\theta}_{s}(t)\right]=\frac{1}{s} f \star \bar{\psi}_{s}(t) .
$$

Thus, in accordance with (21),

$$
W f(t, s)=s \frac{\mathrm{d}}{\mathrm{d} t}\left[f \star \bar{\theta}_{s}(t)\right] .
$$

The wavelet can also take the form of the second derivative of the smoothing function:

$$
\psi(t)=\theta^{\prime \prime}(t) .
$$


Then

$$
W f(t, s)=s^{2} \frac{\mathrm{d}^{2}}{\mathrm{~d} t^{2}}\left[f \star \bar{\theta}_{s}(t)\right] .
$$

In the case of wavelets which are the first derivative of the smoothing function, singular points correspond to the modulus maxima of the wavelet transform; in the case of wavelets which are the second derivative of the smoothing function, singular points correspond to the zero-crossing of the wavelet transform.

Examples of wavelets in the form of the first and second derivatives of the smoothing function are shown in Section 4

The wavelet transform is an effective tool for singularity detection. Mallat (1991), Mallat and Hwang (1992) as well as Mallat and Zhong (1992) show that, if the scale factor $s$ tends to zero, then the points $(t, s)$ on the half-plane $t \in \mathbb{R}, s>0$ corresponding to the modulus maxima of the wavelet transform $W f(t, s)$ represent lines that end in the singular points of the signal.

2.1. Dyadic signal representation. The signal representation in the form of a continuous-time wavelet transform which is a function of two variables (time and scale) is a highly redundant representation. It is proven (Mallat, 2009) that the signal can be reconstructed from a dyadic wavelet transform, in which the scale is not a continuous variable but a countable set of dyadic values: $\left\{s_{k}\right\}_{k \in \mathbb{Z}}$, where $s_{k}=2^{k}$.

The dyadic wavelet transform is defined as follows:

$$
\begin{aligned}
W_{2^{k}} f(u) & =\int_{-\infty}^{+\infty} f(t) \frac{1}{\sqrt{2^{k}}} \psi\left(\frac{t-u}{2^{k}}\right) \mathrm{d} t \\
& =f \star \bar{\psi}_{2^{k}}(u),
\end{aligned}
$$

where $k \in \mathbb{Z}$. This transform is a countable set of functions of one variable (time or space).

In the frequency domain, the dyadic wavelet transform is expressed as

$$
W_{2^{k}} F(j \omega)=\sqrt{2^{k}} F(j \omega) \bar{\Psi}\left(j 2^{k} \omega\right) .
$$

To calculate the wavelet transform at points $\left(n, 2^{k}\right)$, $n, k \in \mathbb{Z}$ (linearly spaced points in time and a dyadic scale), a fast algorithm can be used which is called in French algorithme à trous (Holschneider et al., 1989; Shensa, 1992).

The first step is to define a rectangle $\left[t_{\min }, t_{\max }\right] \times$ $\left[s_{\min }, s_{\max }\right]$ in which the values of the wavelet transform will be calculated. Two rectangles are commonly used: $[0, N-1] \times\left[2, s_{\max }\right]$ or $[0,1) \times\left[s_{\min }, 1\right]$, where $N=2^{K}$. In the case of the rectangle $[0, N-1] \times\left[2, s_{\max }\right]$, the wavelet transform values $W_{2^{k}} f(n)$ are calculated in points $n=0,1, \ldots, N-1$, for $k=1,2, \ldots, K$, where $K=\log _{2} N$. In the case of the rectangle $[0,1) \times$ $\left[s_{\text {min }}, 1\right]$, the wavelet transform values $W_{2^{k}} f(n / N)$ are calculated at points $n=0,1, \ldots, N-1$, for $k=-(K-$ $1),-(K-2), \ldots, 0$.

The application of the à trous algorithm to compute the wavelet transform requires the knowledge the digital representation of the signal $f(t)$ in the finest scale, i.e., scale $s=1$ in the case of window $[0, N-1] \times\left[2, s_{\max }\right]$ or scale $s=1 / N$ in the case of window $[0,1) \times\left[s_{\min }, 1\right]$. From now, on the descriptions of the algorithms will concern the rectangle $[0, N-1] \times\left[2, s_{\max }\right]$ on the plane $0 t s$.

\subsection{Discrete dyadic wavelet transform. Let}

$$
\begin{gathered}
d_{k}[n]=W_{2^{k}} f(n), \\
a_{K}[n]=\int_{-\infty}^{+\infty} f(u) \frac{1}{\sqrt{2^{K}}} \phi\left(\frac{u-n}{2^{K}}\right) \mathrm{d} u \\
=f \star \bar{\phi}_{2^{K}}(n),
\end{gathered}
$$

$k=1,2, \ldots, K$ and $n=0,1, \ldots, N-1$.

The sequence of the numbers

$$
\begin{gathered}
\left.\left\{d_{k}[n]\right\}_{n=0,1, \ldots, N-1}\right\}_{k=1,2, \ldots, K}, \\
\left\{a_{K}[n]\right\}_{n=0,1, \ldots, N-1}
\end{gathered}
$$

is called the discrete dyadic $K$-level wavelet transform of signal $f(t)$.

The wavelet coefficient $d_{k}[n]$ is a measure of the similarity (in the sense of the inner product) of signal $f(t)$ in the surrounding of time instant (a point in space) $t=n$ to the wavelet $\psi\left(t / 2^{k}\right)$ or the value of the signal filtered with a band-pass filter with the impulse response $\bar{\psi}\left(t / 2^{k}\right)$ at $t=n$.

The coefficient $a_{K}[n]$ is a measure of a similarity of signal $f(t)$ in the surrounding of the time instant $t=n$ to the scaling function $\phi\left(t / 2^{K}\right)$ or the value of the signal filtered by a low-pass filter with the impulse response $\bar{\phi}\left(t / 2^{K}\right)$ at $t=n$.

A fast algorithm for computing a discrete dyadic wavelet transform by using digital filtering is described in Section 2.4.

The following section describes how to obtain a discrete-time signal $\left\{a_{0}[n]\right\}_{n \in \mathbb{Z}}$ which is a digital representation of the signal $f(t)$ at the finest scale and forms input to the à trous algorithm for the calculation of a discrete dyadic wavelet transform.

\subsection{Digital representation of the signal at the finest} scale. The sequence of numbers equal to the inner product of signal $f(t)$ and the translation of the scaling function $\phi(t-n)$

$$
a_{0}[n]=\int_{-\infty}^{+\infty} f(t) \phi(t-n) \mathrm{d} t, \quad n \in \mathbb{Z}
$$


is a discrete representation of signal $f(t)$ at scale $2^{0}$. By introducing in the above equation a new variable $u=t-$ $n$, we obtain

$$
a_{0}[n]=\int_{-\infty}^{+\infty} f(n+u) \phi(u) \mathrm{d} u, \quad n \in \mathbb{Z},
$$

showing that the $n$-th sample of the discrete signal is produced by the averaging signal $f(t)$ in the surrounding of the time instant $t=n$, with the scaling function $\phi(u)$ as a weighing function. The sequence $\left(a_{0}[n]\right)$ can also be considered a sample of the signal filtered by the filter with the impulse response equal $\bar{\phi}(t)=\phi(-t)$. Indeed, let the signal $f_{0}(t)$ be the result of filtration. Thus,

$$
f_{0}(t)=\int_{-\infty}^{+\infty} f(u) \bar{\phi}(t-u) \mathrm{d} u,
$$

and then $a_{0}[n]=f_{0}(n)$. The Fourier transform of signal $f_{0}(t)$ is given by

$$
F_{0}(j \omega)=F(j \omega) \bar{\Phi}(j \omega)
$$

2.4. Algorithme à trous. In order to describe the algorithm for computing the discrete dyadic wavelet transform, numerical sequences are introduced and defined as follows:

$$
\begin{aligned}
a_{k}[n] & =\int_{-\infty}^{+\infty} f(u) \frac{1}{\sqrt{2^{k}}} \phi\left(\frac{u-n}{2^{k}}\right) \mathrm{d} u \\
& =f \star \bar{\phi}_{2^{k}}(n), \\
d_{k}[n] & =\int_{-\infty}^{+\infty} f(u) \frac{1}{\sqrt{2^{k}}} \psi\left(\frac{u-n}{2^{k}}\right) \mathrm{d} u \\
& =f \star \bar{\psi}_{2^{k}}(n),
\end{aligned}
$$

$n=0,1, \ldots, N-1$ and $k=1, \ldots, K$.

Let $h_{k}=\left(h_{k}[n]\right), k \geq 0$, be a digital filter with the impulse response created from the impulse response of filter $h=(h[n])$ in such a way that, between each two adjacent coefficients of filter $h, 2^{k}-1$ zeros are inserted, and $\bar{h}_{k}[n]=h_{k}[-n]$; analogically, this is applied to filters $g_{k}$. The coefficients of the discrete dyadic wavelet transform of signal $a_{0}$ can be calculated in the iterative process specified in the following theorem.

Theorem 1. For $k \geq 0$,

$$
\begin{aligned}
& d_{k+1}[n]=a_{k} \star \bar{g}_{k}[n], \\
& a_{k+1}[n]=a_{k} \star \bar{h}_{k}[n] .
\end{aligned}
$$

Proof. The proof of this theorem can be found in the book of Mallat (2009).

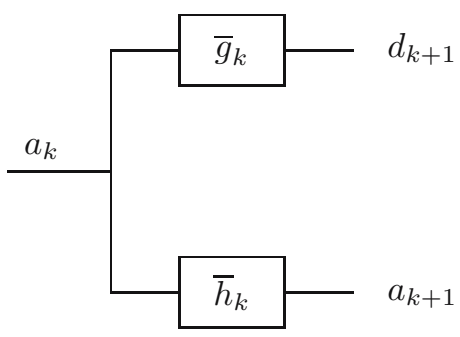

Fig. 1. Filter bank performing one step of the à trous algorithm.

Figure 1 shows a bank of digital filters implementing one step of the discrete dyadic wavelet transform.

The transfer functions of the filters $\bar{g}_{k}$ and $\bar{h}_{k}$ are respectively equal to

$$
\begin{aligned}
& \bar{G}_{k}\left(e^{j \omega}\right)=\bar{G}\left(e^{j 2^{k} \omega}\right), \\
& \bar{H}_{k}\left(e^{j \omega}\right)=\bar{H}\left(e^{j 2^{k} \omega}\right) .
\end{aligned}
$$

\section{Box splines}

The starting point for the construction of a box spline is defining the gate function (Unser, 1999; Press et al., 2007; Boor, 1978)

$$
b(t)=\left\{\begin{array}{cc}
1, & 0 \leq t<1, \\
0, & \text { otherwise. }
\end{array}\right.
$$

The Fourier transform of the gate function is

$$
\begin{aligned}
B(j \omega) & =\frac{1-e^{-j \omega}}{j \omega} \\
& =e^{-j \omega / 2} \frac{\sin (\omega / 2)}{\omega / 2} .
\end{aligned}
$$

The gate function $b(t)$ is a box spline of zero degree $b_{0}(t)$. The box spline of $m$ degree is defined recursively as a convolution of the gate function $b(t)$ with the box spline of $m-1$ degree, thus $b_{m}(t)=b_{m-1} \star b(t), m=1,2, \ldots$. The support of the box spline of $m$ degree is included in interval $[0, m+1]$.

The convolution in the time domain corresponds to multiplication in the frequency domain of Fourier transforms, so the Fourier transform of the box spline of the $m$-th degree is

$$
B_{m}(j \omega)=e^{-j(m+1) \omega / 2}\left[\frac{\sin (\omega / 2)}{\omega / 2}\right]^{m+1} .
$$


The box spline of degree $m$ can be calculated by using the box splines of degree $m-1$,

$$
b_{m}(t)=\left[t b_{m-1}(t)+(m+1-t) b_{m-1}(t-1)\right] / m .
$$

The derivative of box spline of degree $m$ can be calculated by using the box spline of degree $m-1$,

$$
b_{m}^{\prime}(t)=b_{m-1}(t)-b_{m-1}(t-1)
$$

The integer translations of box splines $b_{m}(t-n), n \in \mathbb{Z}$, are not orthogonal to one another. Thus

$$
\int_{-\infty}^{+\infty} b_{m}(t-n) b_{m}(t-k) \mathrm{d} t \neq \delta[n-k] .
$$

There is a procedure of orthogonalization; however, splines received as a result do not have a compact support but decay quickly (exponentially).

3.1. Box spline of the third degree. The box spline of degree $3 b_{3}(t)$ has a support equal to interval $[0,4]$.

On each of the subintervals $[k, k+1], 0 \leq k \leq$ 3 , the value of the function is determined by another polynomial of the third degree, but a connection of different polynomials in the internal nodes, i.e., at points $t=1,2,3$, is twice continuously differentiable.

Table 1 shows polynomials defining the box spline of the third degree, and Fig. 2 presents the shape of the function.

Table 1. Polynomials describing the box spline of the third degree $b_{3}(t)$.

\begin{tabular}{|r||l|}
\hline Interval & $b_{3}(t)$ \\
\hline \hline$t<0$ & 0 \\
\hline $0 \leq t<1$ & $\frac{1}{6} t^{3}$ \\
\hline $1 \leq t<2$ & $-\frac{1}{2} t^{3}+2 t^{2}-2 t+\frac{2}{3}$ \\
\hline $2 \leq t<3$ & $-\frac{1}{2}(4-t)^{3}+2(4-t)^{2}-2(4-t)+\frac{2}{3}$ \\
\hline $3 \leq t<4$ & $\frac{1}{6}(4-t)^{3}$ \\
\hline$t \geq 4$ & 0 \\
\hline
\end{tabular}

The Fourier transform of the cubic box spline is

$$
B_{3}(j \omega)=e^{-j 2 \omega}\left[\frac{\sin (\omega / 2)}{\omega / 2}\right]^{4} .
$$

\section{Scaling function and wavelet in the form of a cubic box spline}

The scaling function is expressed as a box spline function of the third degree shifted to the left by two units. Thus

$$
\phi(t)=b_{3}(t+2)
$$

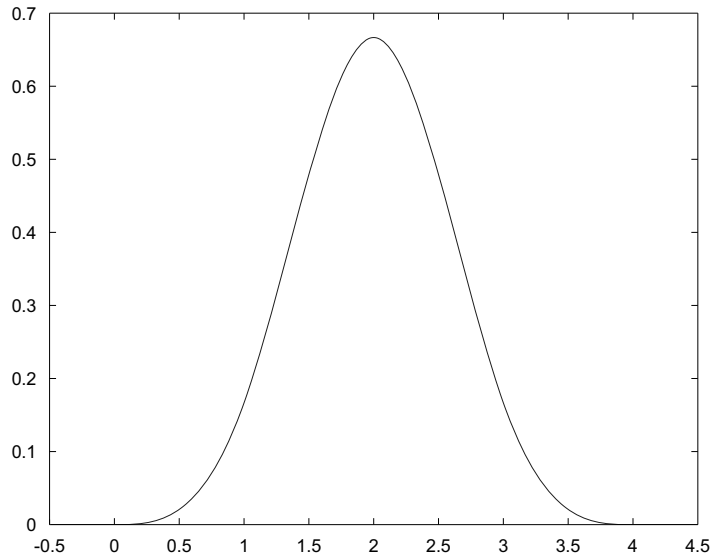

Fig. 2. Box spline of the third degree $b_{3}(t)$.

Figure 3 shows the graph of the scaling function and Table 2 the polynomials describing that function.

The Fourier transform of the scaling function is equal

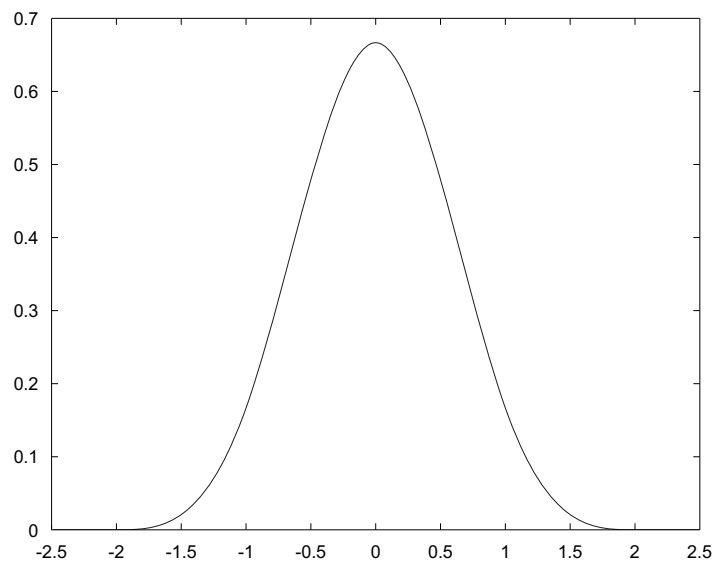

Fig. 3. Scaling function $\phi(t)$ in the form of translation of the cubic box spline.

Table 2. Polynomials describing the scaling function $\phi(t)$ in the form of the cubic box spline.

\begin{tabular}{|r||l|}
\hline Interval & $\phi(t)$ \\
\hline \hline$t<-2$ & 0 \\
\hline$-2 \leq t<-1$ & $(t+2)^{3} / 6$ \\
\hline$-1 \leq t<0$ & $-t^{3} / 2-t^{2}+2 / 3$ \\
\hline $0 \leq t<1$ & $t^{3} / 2-t^{2}+2 / 3$ \\
\hline $1 \leq t<2$ & $(2-t)^{3} / 6$ \\
\hline$t \geq 2$ & 0 \\
\hline
\end{tabular}




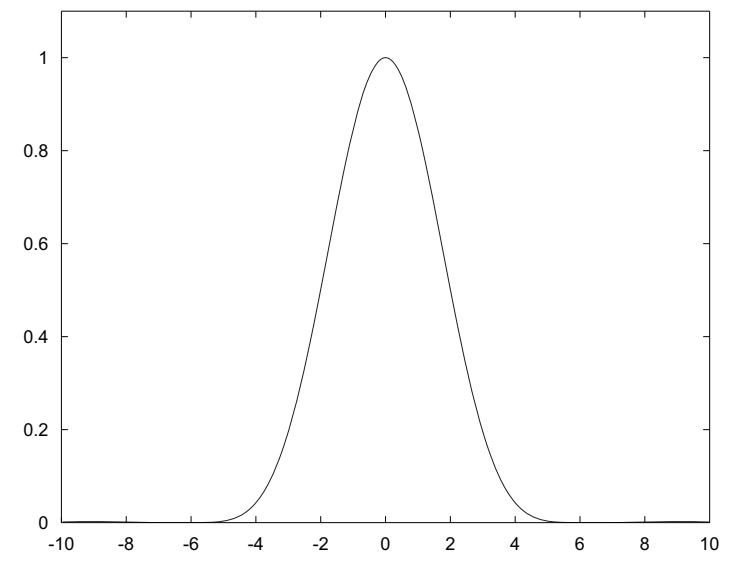

Fig. 4. Magnitude of the Fourier transform of the scaling function $\phi(t)$ in the form of a cubic box spline.

to

$$
\Phi(j \omega)=\left[\frac{\sin (\omega / 2)}{\omega / 2}\right]^{4} .
$$

Figure 4 shows the magnitude of the Fourier transform of the scaling function in the form of a cubic box spline. The transfer function of filter $h$ corresponding to the scaling function $\phi(t)$ can be calculated from the dilation equation expressed in the frequency domain (13),

$$
H\left(e^{j \omega}\right)=\sqrt{2} \frac{\Phi(j 2 \omega)}{\Phi(j \omega)} .
$$

The transfer function of filter $h$ corresponding to the scaling function as a cubic box spline is

$$
\begin{aligned}
H\left(e^{j \omega}\right)= & \sqrt{2}\left(\frac{\sin \omega}{\omega}\right)^{4}\left[\frac{\sin (\omega / 2)}{\omega / 2}\right]^{-4} \\
= & \sqrt{2}\left(\cos \frac{\omega}{2}\right)^{4} \\
= & \frac{\sqrt{2}}{16}\left(e^{j 2 \omega}+4 e^{j \omega}+6\right. \\
& \left.+4 e^{-j \omega}+e^{-j 2 \omega}\right) .
\end{aligned}
$$

The magnitude of the transfer function of filter $h$ is shown in Fig. 5. The coefficients of filter $h$ are summarized in Table 3

Table 3. Coefficients of the lowpass filter $h[n]$ corresponding to the scaling function $\phi(t)$ as a cubic box spline.

\begin{tabular}{|c||c|c|c|c|c|}
\hline$n$ & -2 & -1 & 0 & 1 & 2 \\
\hline \hline$h[n]$ & $\frac{\sqrt{2}}{16}$ & $\frac{\sqrt{2}}{4}$ & $\frac{3 \sqrt{2}}{8}$ & $\frac{\sqrt{2}}{4}$ & $\frac{\sqrt{2}}{16}$ \\
\hline
\end{tabular}

The wavelet $\psi(t)$ can be constructed based on Eqn. (14) by first selecting the high-pass filter $g$. The shortest high-pass filter is the Haar one, for which the impulse response coefficients are given in Table 4 and the transfer function is equal to

$$
\begin{aligned}
G\left(e^{j \omega}\right) & =-\frac{\sqrt{2}}{2}+\frac{\sqrt{2}}{2} e^{-j \omega} \\
& =-j \sqrt{2} e^{-j \frac{\omega}{2}} \sin \frac{\omega}{2} .
\end{aligned}
$$

Table 4. Coefficients of the high-pass filter $g[n]$ corresponding to the wavelet $\psi(t)$ in the form of the cubic spline 57 .

\begin{tabular}{|c||c|c|}
\hline$n$ & 0 & 1 \\
\hline \hline$g[n]$ & $-\frac{\sqrt{2}}{2}$ & $\frac{\sqrt{2}}{2}$ \\
\hline
\end{tabular}

The wavelet corresponding to this filter is equal to

$$
\psi(t)=-\phi(2 t)+\phi(2 t-1) .
$$

From (52), we obtain $\phi(2 t)=b_{3}(2 t+2)$ and $\phi(2 t-1)=$ $b_{3}(2 t+1)$, so

$$
\psi(t)=-b_{3}(2 t+2)+b_{3}(2 t+1) .
$$

It is easy to see that the support of the wavelet is the interval $[-1,1.5]$.

By introducing a new variable $\tau=2 t+2$, Eqn. (58) takes the form of

$$
-\psi\left(\frac{\tau-2}{2}\right)=b_{3}(\tau)-b_{3}(\tau-1) .
$$

The right hand-side of the above equation, according to (49), is the derivative of $b_{4}(\tau)$, so

$$
\psi\left(\frac{\tau-2}{2}\right)=-\frac{\mathrm{d}}{\mathrm{d} \tau} b_{4}(\tau) .
$$

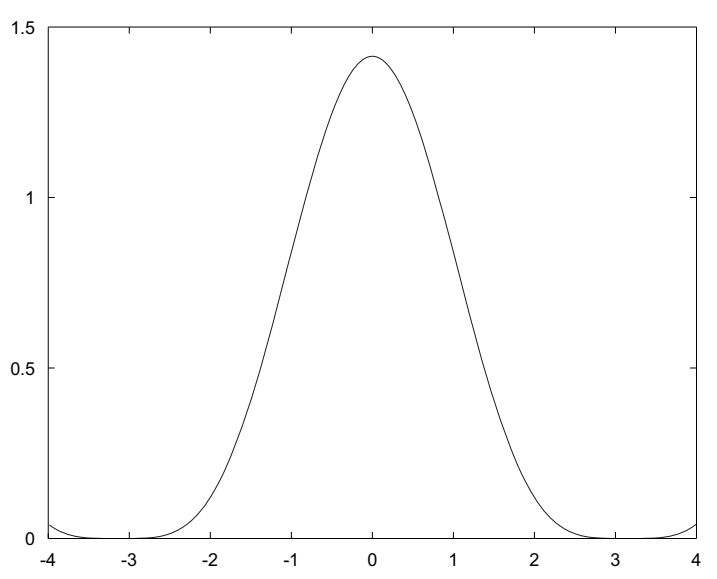

Fig. 5. Magnitude of the transfer function of filter $h$ corresponding to the scaling function as a cubic box spline. 
Going back to the variable $t$, we obtain

$$
\psi(t)=-\frac{\mathrm{d}}{\mathrm{d} t}\left[\frac{1}{2} b_{4}(2 t+2)\right],
$$

which means that the smoothing function is equal to

$$
\theta(t)=\frac{1}{2} b_{4}(2 t+2)
$$

and $\psi(t)=-\theta^{\prime}(t)$.

The Fourier transform of the wavelet is calculated using the wavelet equation (14),

$$
\begin{aligned}
\Psi(j \omega) & =\frac{1}{\sqrt{2}} G\left(e^{j \frac{\omega}{2}}\right) \Phi(j \omega / 2) \\
& =\frac{-j \omega}{4} e^{-j \frac{\omega}{4}}\left[\frac{\sin (\omega / 4)}{\omega / 4}\right]^{5} .
\end{aligned}
$$

The transfer function of filter $g$ expressed by the formula (56) has one zero for $\omega=0$, so the corresponding wavelet has one vanishing moment.

Table 5. Polynomials describing the wavelet $\psi(t)$ in the form of a cubic spline corresponding to filter $g$ with coefficients given in Table 4

\begin{tabular}{|r||l|}
\hline Interval & $\psi(t)$ \\
\hline \hline$t<-1$ & 0 \\
\hline$-1 \leq t<-0.5$ & $-\frac{4}{3}(1+t)^{3}$ \\
\hline$-0.5 \leq t<0$ & $\frac{16}{3} t^{3}+6 t^{2}+t-\frac{1}{2}$ \\
\hline $0 \leq t<0.5$ & $-8 t^{3}+6 t^{2}+t-\frac{1}{2}$ \\
\hline $0.5 \leq t<1$ & $\frac{16}{3} t^{3}-14 t^{2}+11 t-\frac{13}{6}$ \\
\hline $1 \leq t<1.5$ & $\frac{4}{3}\left(\frac{3}{2}-t\right)^{3}$ \\
\hline$t \geq 1.5$ & 0 \\
\hline
\end{tabular}

Table 6. Smoothing function $\theta(t)$ in the form of a scaled and translated box spline of the fourth degree.

\begin{tabular}{|r||l|}
\hline Interval & $\theta(t)$ \\
\hline \hline$t<-1$ & 0 \\
\hline$-1 \leq t<-0.5$ & $-\frac{1}{3}(1+t)^{4}$ \\
\hline$-0.5 \leq t<0$ & $-\frac{4}{3} t^{4}-2 t^{3}-\frac{1}{2} t^{2}+\frac{1}{2} t+\frac{11}{48}$ \\
\hline $0 \leq t<0.5$ & $2 t^{4}-2 t^{3}-\frac{1}{2} t^{2}+\frac{1}{2} t+\frac{11}{48}$ \\
\hline $0.5 \leq t<1$ & $-\frac{4}{3} t^{4}+\frac{14}{3} t^{3}-\frac{11}{2} t^{2}+\frac{13}{6} t+\frac{1}{48}$ \\
\hline $1 \leq t<1.5$ & $\frac{1}{3}(1.5-t)^{4}$ \\
\hline$t \geq 1.5$ & 0 \\
\hline
\end{tabular}

An alternative wavelet $\psi(t)$ can be constructed by selecting the high-pass filter $g$ with three impulse response coefficients given in Table 7 and the transfer function

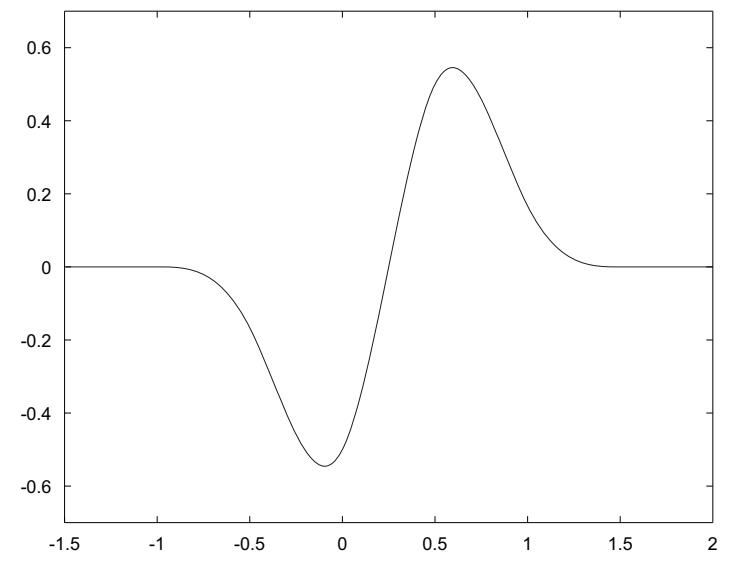

Fig. 6. Wavelet $\psi(t)$ in the form of a cubic spline corresponding to filter $g$ with coefficients given in Table 4

equal to

$$
\begin{aligned}
G\left(e^{j \omega}\right) & =\frac{\sqrt{2}}{4} e^{j \omega}-\frac{\sqrt{2}}{2}+\frac{\sqrt{2}}{4} e^{-j \omega} \\
& =-\sqrt{2}\left(\sin \frac{\omega}{2}\right)^{2} .
\end{aligned}
$$

Table 7. Coefficients of the high-pass filter $g[n]$ corresponding to the wavelet $\psi(t)$ in the form of a spline function of the third degree 65.

\begin{tabular}{|c||c|c|c|}
\hline$n$ & -1 & 0 & 1 \\
\hline \hline$g[n]$ & $\frac{\sqrt{2}}{4}$ & $-\frac{\sqrt{2}}{2}$ & $\frac{\sqrt{2}}{4}$ \\
\hline
\end{tabular}

The wavelet corresponding to this filter is equal to

$$
\psi(t)=\frac{1}{2} \phi(2 t+1)-\phi(2 t)+\frac{1}{2} \phi(2 t-1),
$$

and has support $[-1.5,1.5]$. Filter $g$ with the transfer function 64 has two zeros for $\omega=0$, so the corresponding wavelet has two vanishing moments. In Fig. 10, it can be seen that the wavelet has three local extremes. The Fourier transform of the wavelet is equal to

$$
\Psi(j \omega)=\frac{(j \omega)^{2}}{4}\left[\frac{\sin (\omega / 4)}{\omega / 4}\right]^{6}
$$

The expression

$$
\frac{1}{2}\left[\frac{\sin (\omega / 4)}{\omega / 4}\right]^{6}
$$

is, in accordance with 477, the Fourier transform of $b_{5}\left(2\left(t+\frac{3}{2}\right)\right)$. The value $(j \omega)^{2}$ in the Fourier transform corresponds to the second derivative of function 


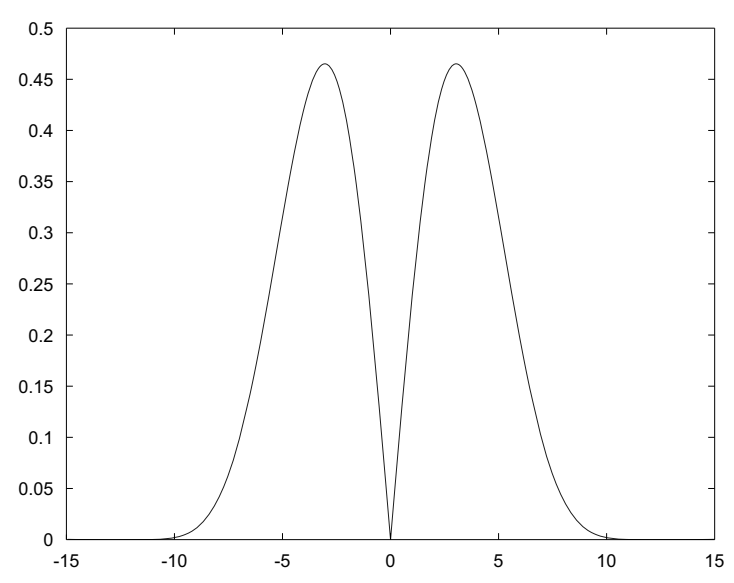

Fig. 7. Magnitude of the Fourier transform of the wavelet $\psi(t)$ in the form of a cubic spline corresponding to filter $g$ with coefficients given in Table 4

$b_{5}\left(2\left(t+\frac{3}{2}\right)\right)$. Thus, the wavelet

$$
\psi(t)=\frac{\mathrm{d}^{2}}{\mathrm{~d} t^{2}}\left\{\frac{1}{2} b_{5}\left[2\left(t+\frac{3}{2}\right)\right]\right\},
$$

and the smoothing function is

$$
\theta(t)=\frac{1}{2} b_{5}(2 t+3)
$$

The discussed wavelet is similar to the Mexican hat wavelet, described by

$$
\psi(t)=\frac{2}{\pi^{1 / 4} \sqrt{3}}\left(t^{2}-1\right) e^{-t^{2} / 2}
$$

and shown in Fig. 12

The Mexican hat wavelet is the second derivative of the Gaussian function. The Gaussian function is the result of convolution of an infinite number of gate functions, i.e., $\lim _{m \rightarrow+\infty} b_{m}(t)$; it belongs to the space of function $C^{\infty}$ and, as the scale factor tends to zero, the Gaussian function tends to the Dirac distribution $\delta(t)$. The whole axis $(-\infty,+\infty)$ is the support of the Gaussian function and the Mexican hat wavelet. The Mexican hat wavelet was the first one used for multiscale filtering (Witkin, 1983; 1984) and detection of signal singularities (Mallat, 1991).

If there is a need for having synthesis filters (reconstruction filters), then a low-pass filter of the synthesis can be assumed equal to the low-pass filter analysis, i.e., $\tilde{h}=h$, as a consequence of the synthesis scaling function $\widetilde{\phi}(t)=\phi(t)$. The transfer function of the high-pass synthesis filter $\tilde{g}$ can be calculated from the conditions of a perfect signal reconstruction (Mallat, 2009;

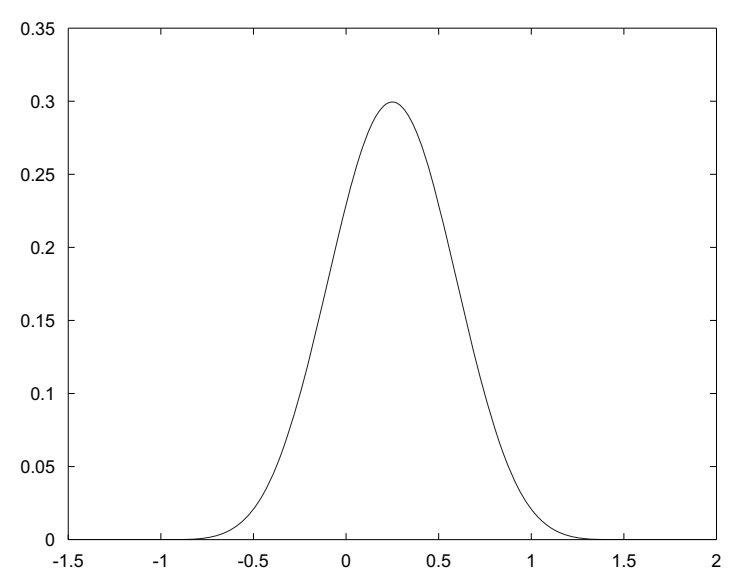

Fig. 8. Smoothing function $\theta(t)$ in the form of a scaled and translated box spline of the fourth degree.

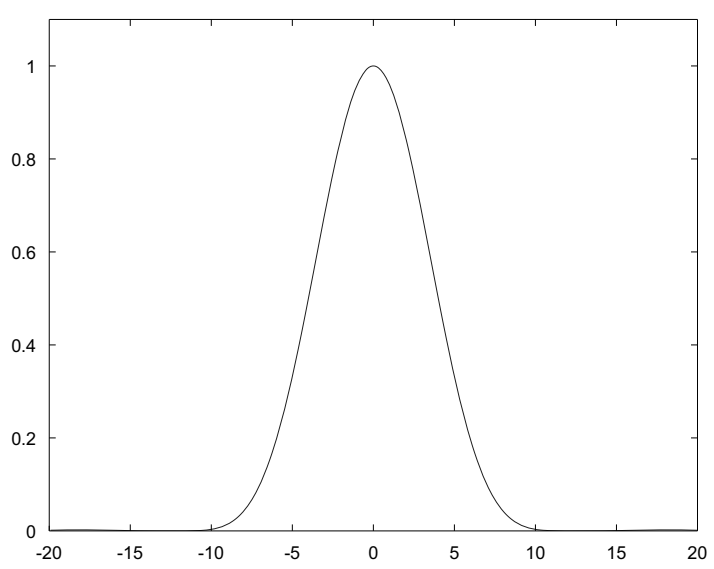

Fig. 9. Magnitude of the Fourier transform of the smoothing function $\theta(t)$ as a scaled and translated box spline of the fourth degree.

Rakowski, 2003) expressed by

$$
\widetilde{H}\left(e^{j \omega}\right) H^{*}\left(e^{j \omega}\right)+\widetilde{G}\left(e^{j \omega}\right) G^{*}\left(e^{j \omega}\right)=2,
$$

from which

$$
\widetilde{G}\left(e^{j \omega}\right)=\frac{2-\left|H\left(e^{j \omega}\right)\right|^{2}}{G^{*}\left(e^{j \omega}\right)} .
$$

Having the transfer function of the filter $\widetilde{G}\left(e^{j \omega}\right)$, one can calculate the coefficients of the impulse response $\tilde{g}[n]$, and the synthesis wavelet $\widetilde{\psi}(t)$ is calculated using the wavelet equation (14). Different synthesis wavelet filters calculated with the FFT algorithm are presented by Zhao et al. (2013). 


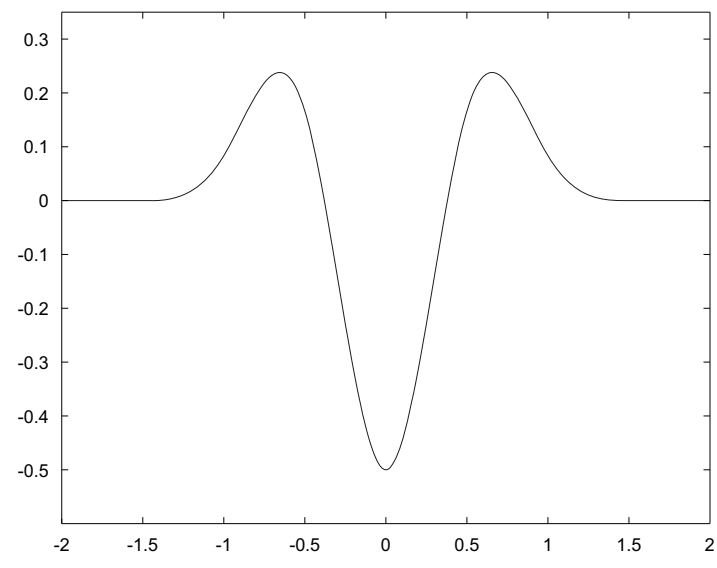

Fig. 10. Wavelet $\psi(t)$ in the form of a cubic spline corresponding to filter $g$ with coefficients given in Table 7

\section{Scale-frequency analysis of a dyadic wavelet transform}

Multiscale signal decomposition by a dyadic wavelet transform can be seen as a band decomposition of a signal in the frequency domain.

Figure 13 shows a filter configuration carrying $K$-level discrete dyadic decomposition of signal $a_{0}$. It follows from it that the Fourier transform of signals $d_{k}$, $k=1,2, \ldots, K$, can be expressed as

$$
\begin{gathered}
D_{1}\left(e^{j \omega}\right)=A_{0}\left(e^{j \omega}\right) \bar{G}_{0}\left(e^{j \omega}\right), \\
D_{k}\left(e^{j \omega}\right)=A_{0}\left(e^{j \omega}\right) \bar{G}_{k-1}\left(e^{j \omega}\right) \prod_{l=0}^{k-2} \bar{H}_{l}\left(e^{j \omega}\right),
\end{gathered}
$$

$k=2,3, \ldots, K$. Defining the equivalent filter transfer functions at scales $2^{k}, k=1,2, \ldots, K$, as

$$
P_{k}\left(e^{j \omega}\right)=D_{k}\left(e^{j \omega}\right) / A_{0}\left(e^{j \omega}\right)
$$

and taking into account (44) and (45), we obtain

$$
\begin{gathered}
P_{1}\left(e^{j \omega}\right)=\bar{G}\left(e^{j \omega}\right), \\
P_{k}\left(e^{j \omega}\right)=\bar{G}\left(e^{j 2^{k-1} \omega}\right) \prod_{l=0}^{k-2} \bar{H}\left(e^{j 2^{l} \omega}\right),
\end{gathered}
$$

$k=2,3, \ldots, K$.

In the case of filters $\bar{h}, \bar{g}$ with real impulse response coefficients, the transfer functions take the form of

$$
\begin{gathered}
P_{1}\left(e^{j \omega}\right)=G^{*}\left(e^{j \omega}\right), \\
P_{k}\left(e^{j \omega}\right)=G^{*}\left(e^{j 2^{k-1} \omega}\right) \prod_{l=0}^{k-2} H^{*}\left(e^{j 2^{l} \omega}\right),
\end{gathered}
$$

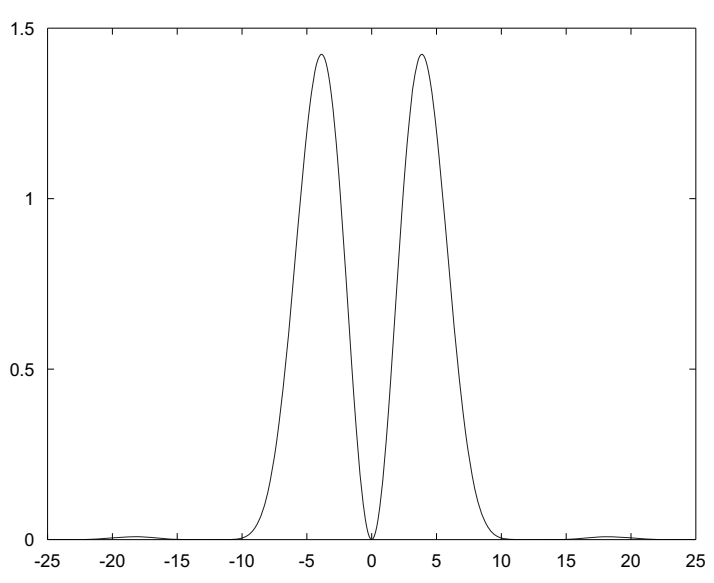

Fig. 11. Magnitude of the Fourier transform of the wavelet $\psi(t)$ in the form of a cubic spline corresponding to filter $g$ with coefficients given in Table 7

5.1. Frequency characteristics of the equivalent cubic filters. The starting point for the construction of the frequency characteristics of the equivalent cubic filters $P_{k}, k=2,3, \ldots, K$, is a transfer function of the filter associated with the scaling function (55),

$$
H\left(e^{j \omega}\right)=\sqrt{2}\left(\cos \frac{\omega}{2}\right)^{4},
$$

and a transfer function of the filter associated with a wavelet.

5.1.1. Wavelet as the first derivative of a smoothing function. In this case, the smoothing function is a box spline of the fourth degree and the wavelet is a cubic box spline. A transfer function of the filter corresponding to the wavelet as the first derivative of a smoothing function, according to (56), is equal to

$$
G\left(e^{j \omega}\right)=-j \sqrt{2} e^{-j \frac{\omega}{2}} \sin \frac{\omega}{2} .
$$

Transfer functions of equivalent filters are obtained after inserting (80) and (81) into (78) and (79). Figure 14 shows the magnitudes of the filter transfer functions at scales $2^{2}, 2^{3}, 2^{4}, 2^{5}$. Table 8 presents $3 \mathrm{~dB}$ pass-bands of these filters.

5.1.2. Wavelet as the second derivative of a smoothing function. In this case, the smoothing function is a box spline of the fifth degree and the wavelet is a cubic spline. A transfer function of the filter associated with the wavelet in the form of the second derivative of a smoothing function, according to (64), is equal to

$$
G\left(e^{j \omega}\right)=-\sqrt{2}\left(\sin \frac{\omega}{2}\right)^{2} .
$$




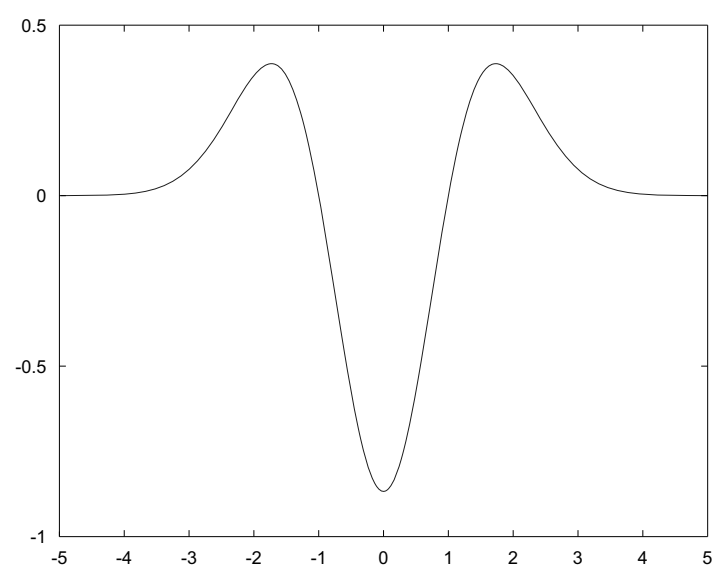

Fig. 12. Mexican hat wavelet.

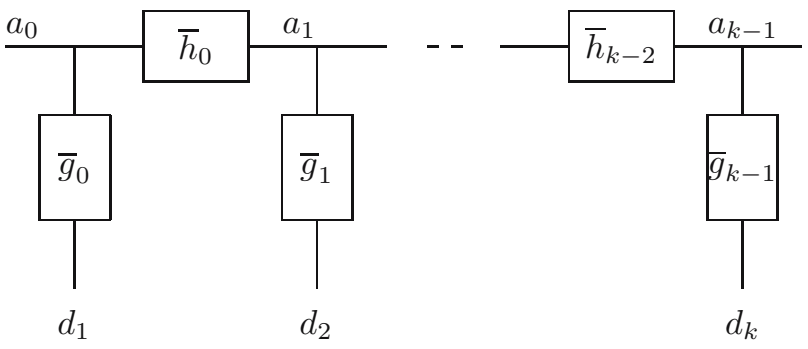

Fig. 13. Filter bank implementing the à trous algorithm.

Transfer functions of the equivalent filters are obtained after inserting (80) and (82) into (78) and (79). Figure 15] shows magnitudes of the transfer functions of the equivalent filters at scales $2^{2}, 2^{3}, 2^{4}, 2^{5}$. Table 9 shows $3 \mathrm{~dB}$ passband of these filters.

\section{Regularity and singularity of a signal}

The formal definition of the singularity of a signal is associated with the Lipschitz exponent and Lipschitz regularity, explained below. If the signal $f(t)$ is differentiable, then the number of the derivatives is a measure of its smoothness. Let the signal $f(t)$ have the Fourier transform $F(j \omega)$ and be $m$-times differentiable. The Fourier transform of the $m$-th derivative of the signal is $(j \omega)^{m} F(j \omega)$. If the value of $\alpha$ is in the interval $m \leq \alpha<m+1$ and such that

$$
\int_{-\infty}^{+\infty}|F(j \omega)|\left(1+|\omega|^{\alpha}\right) \mathrm{d} \omega<+\infty
$$
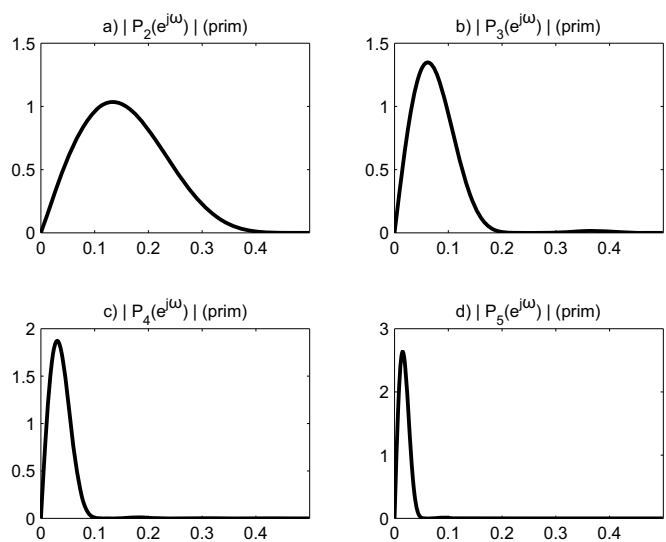

Fig. 14. Magnitudes of the filter transfer functions associated with the cubic box wavelet in the form of the first derivative of the smoothing function at scales $2^{2}, 2^{3}$, $2^{4}, 2^{5}$.

Table 8. $3 \mathrm{~dB}$ pass bands of equivalent filters $P_{k}\left(e^{j \omega}\right)$ at scales $s=2^{k}, k=1,2,3,4,5$

\begin{tabular}{|c|c|r|}
\hline Scale & \multicolumn{2}{|c|}{ Passband $3 \mathrm{~dB}[\mathrm{rad}]$} \\
\hline \hline$s=2^{1}$ & 0.2500 & 0.5000 \\
\hline$s=2^{2}$ & 0.0654 & 0.2129 \\
\hline$s=2^{3}$ & 0.0303 & 0.0986 \\
\hline$s=2^{4}$ & 0.0156 & 0.0488 \\
\hline$s=2^{5}$ & 0.0078 & 0.0234 \\
\hline
\end{tabular}

then it is said that the signal $f(t)$ is uniformly Lipschitz with exponent $\alpha$. If the supremum of values $\alpha$ satisfies the above condition of convergence of the integral, then the supremum is a measure of the global regularity of the signal. The notion of regularity is an extension of the concept of smoothness measured by the number of derivatives. For example, the scaling functions corresponding to the $9 / 7$ low-pass filters (Fig. 16) used in the JPEG2000 image compression standard (Skodras et al., 2001) have Lipschitz exponents equal respectively to 1.07 and 1.70 (Unser and Blu, 2003).

The Fourier transform of the signal does not allow determination of the local regularity of the signal, i.e., the regularity at a point and its surrounding. Point regularity in terms of the Lipschitz exponent is associated with estimation of the error of signal approximation by the Taylor polynomial.

Let the signal $f(t)$ have $m$ continuous derivatives. The Taylor polynomial

$$
p_{v}(t)=f(v)+\sum_{k=1}^{m} \frac{f^{(k)}(t)}{k !}(t-v)^{k}
$$



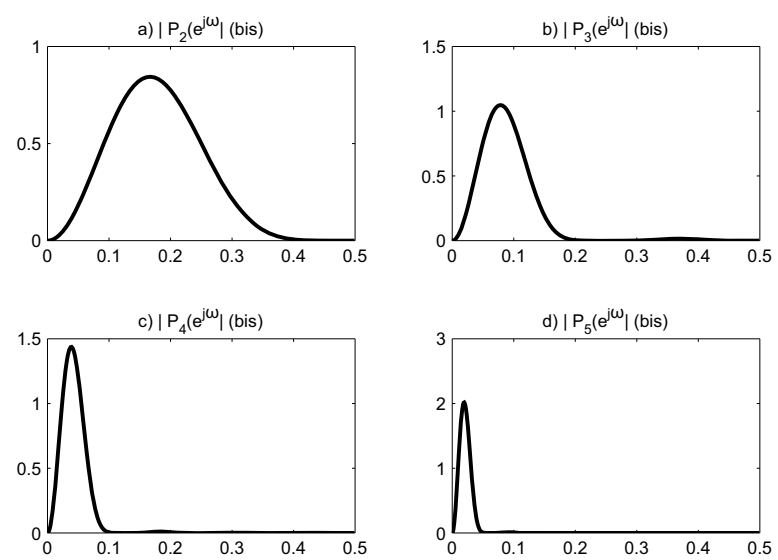
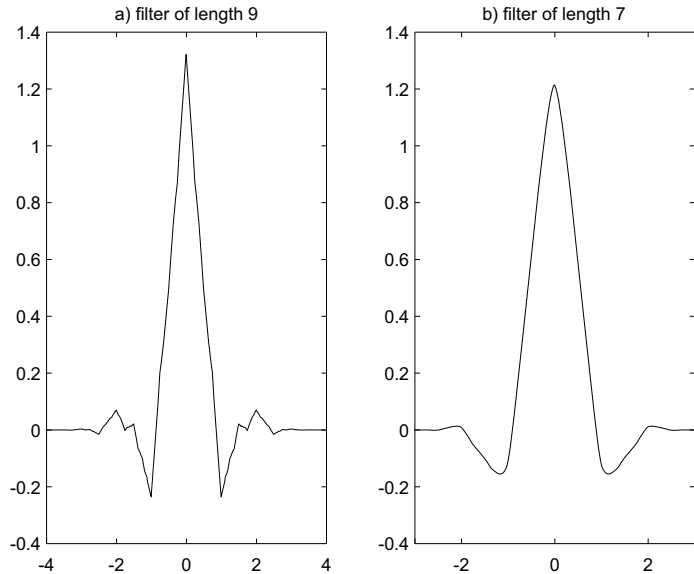

Fig. 15. Magnitudes of the transfer functions of the equivalent filters associated with the cubic wavelet as the second derivative of the smoothing function at scales $2^{2}, 2^{3}$, $2^{4}, 2^{5}$.

Table 9. $3 \mathrm{~dB}$ pass bands of equivalent filters $P_{k}\left(e^{j \omega}\right)$ at scales $s=2^{k}, k=1,2,3,4,5$.

\begin{tabular}{|r|c|c|}
\hline scale & \multicolumn{2}{|c|}{$3 \mathrm{~dB}$ band [rad] } \\
\hline \hline$s=2^{1}$ & 0.3184 & 0.5000 \\
\hline$s=2^{2}$ & 0.1045 & 0.2344 \\
\hline$s=2^{3}$ & 0.0498 & 0.1104 \\
\hline$s=2^{4}$ & 0.0244 & 0.0547 \\
\hline$s=2^{5}$ & 0.0127 & 0.0264 \\
\hline
\end{tabular}

approximates the signal $f(t)$ in the neighborhood of point $v$ with the error

$$
e_{v}(t)=f(t)-p_{v}(t)
$$

The Lipschitz exponent estimates the approximation error and determines the regularity of the signal in the following way (Mallat, 2009):

- At a point the signal $f(t)$ has $v$ the Lipschitz exponent $\alpha \geq 0$ if there exist a constant $K>0$ and polynomial $p_{v}(t)$ of degree $m=\lfloor\alpha\rfloor$ such that, for each $t \in \mathbb{R}$,

$$
\left|f(t)-p_{v}(t)\right| \leq K|t-v|^{\alpha} .
$$

- The signal $f(t)$ is uniformly Lipschitz $\alpha$ into interval $[a, b]$ if it satisfies the Lipschitz condition (86) for all $v \in[a, b]$ with a constant $K$ independent of $v$.

- The Lipschitz regularity of the signal $f(t)$ at a point $v$ or in the interval $[a, b]$ is defined by the supremum of the values $\alpha$ such that $f(t)$ has Lipschitz exponent $\alpha$.
Fig. 16. Scaling functions corresponding to 9/7 biorthogonal filters.

If $0 \leq \alpha<1$, then the signal is non-differentiable at the point $v$ and the Lipschitz condition is expressed by the following inequality:

$$
|f(t)-f(v)| \leq K|t-v|^{\alpha} .
$$

A signal that is bounded but non-continuous at a point $v$ has the Lipschitz exponent equal to 0 . If $0<\alpha<$ 1 , then the signal at the point $v$ is singular. If $\alpha \geq 1$, then $f^{(p)}(t)$, where $p=\lfloor\alpha\rfloor$ has the Lipschitz exponent equal to $\alpha-p$, and if $\alpha-p>0$, then that value is a measure of the singularity of the $p$-th derivative of the signal $f(t)$.

A wavelet transform of a signal, calculated with a wavelet that has enough vanishing moments is equal to the wavelet transform of the error approximation (85). Let the vanishing moment number $p$ of the wavelet be no less than the Lipschitz exponent $\alpha$, i.e., $p \geq \alpha$. Thus,

$$
\begin{aligned}
W f(t, s) & =W p_{v}(t, s)+W e_{v}(t, s) \\
& =W e_{v}(t, s),
\end{aligned}
$$

because the wavelet is orthogonal to $p_{v}(t)$. It follows that, if $0 \leq \alpha \leq 1$, then a wavelet with one vanishing moment can be used; if $1<\alpha \leq 2$, then a wavelet with two vanishing moments should be used. Among all the singular points, the so-called isolated singularities stand out. A point $v$ is an isolated singular point if the maximum values of the wavelet transform modulus at a scale $s$ less than a certain scale $s_{0}$ are located in a cone

$$
|t-v| \leq C s
$$

where $C$ is a certain positive constant. Mallat (2009) shows that in the case of the isolated singular points there exists a constant $A>0$ such that

$$
|W f(t, s)| \leq A s^{\alpha+1 / 2} .
$$


An equivalent form can be obtained by applying a logarithm on both sides of the inequality

$$
\log _{2}|W f(t, s)| \leq(\alpha+1 / 2) \log _{2} s+\log _{2} A,
$$

from which it follows that $\alpha+1 / 2$ is the maximum slope of a straight line $y=r x+q$, where $x=\log _{2} s$ and $y=$ $\log _{2}|W f(t, s)|$.

\section{Numerical results}

Below are two examples illustrating the use of a discrete dyadic wavelet transform and cubic wavelets for detection of slopes (edges) of the signal.

Example 1. Figure 17 presents an artificial signal $f[n]$ of the length of 512 samples containing different slopes. The first two slopes are respectively the leading edge and the trailing edge of the rectangular pulse; the next pulse is a Dirac delta; the further pulse contains step-and cubic slopes; the one but last pulse contains a slope sigmoidal, and the last slope is exponential. A five-level discrete dyadic wavelet decomposition of the signal was computed using the à trous algorithm with a cubic box spline as the scaling function and the wavelet as the first derivative of the smoothing function as shown in Fig. 6 , The low-pass filter coefficients associated with the scaling function are shown in Table 3, whereas the high-pass filter coefficients associated with the wavelet are presented in Table 4. Since the wavelet used is the first derivative of

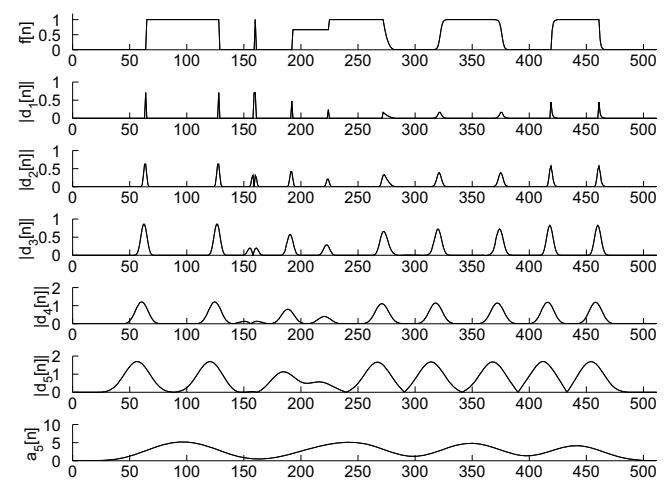

Fig. 17. Example of a digital signal $f[n]$, a modulus of wavelet coefficients $\left|d_{1}[n]\right|,\left|d_{2}[n]\right|,\left|d_{3}[n]\right|,\left|d_{4}[n]\right|,\left|d_{5}[n]\right|$ and a smoothed signal $a_{5}[n]$ of a five-level dyadic decomposition using digital filters from Tables 3 and 4

the smoothing function, the local modulus maxima of the wavelet coefficients $d_{k}[n]$ indicate the singular points of the signal. Noticing that the high-pass filter associated with the wavelet in the form of the first derivative of the smoothing function is not symmetric or antisymmetric at zero (see Table 4), signals $d_{k}[n]$ are shifted to the left (according to Eqns. (79) and (81)) by $2^{k-2}$ samples. Therefore, the position of the modulus of local extrema in the signals $d_{k}[n]$ is shifted to the left by $2^{k-2}$ samples with respect to singular points of the signal (smoothed signal inflection points).

Figure 17 shows that at fine scales (signals $d_{1}[n]$, $\left.d_{2}[n]\right)$ the wavelet coefficients corresponding to smooth slopes are much smaller than those corresponding to sharp slopes. However, in coarse scales (signals $d_{4}[n], d_{5}[n]$ ), coefficients are comparable. Owing to that fact, the multiscale analysis allows us to identify singular points at coarse scales and facilitates accurate location of these points at fine scales. Figure 18 shows a signal from Fig.17 with an added noise. Decomposition of a noisy signal was performed using the same filters that were used for the signal decomposition in Fig. 17 Figure 18 shows that the wavelet analysis of a noisy signal at fine scales practically does not allow detection of singular points, and the multiscale analysis, due to filtration of noise at coarse scales, allows identification and location of these points.

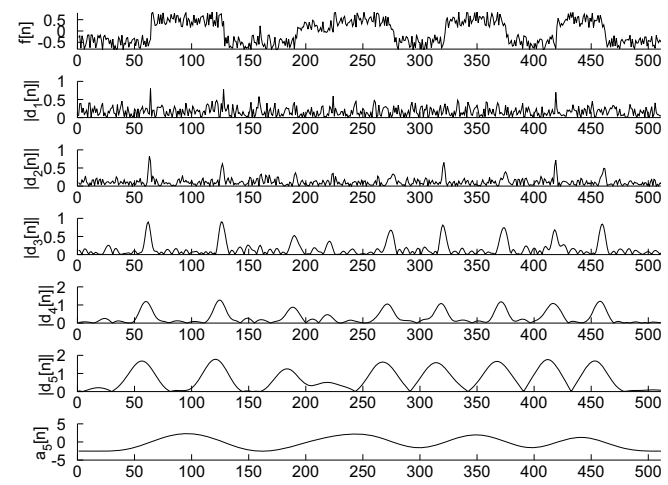

Fig. 18. Example of a signal $f[n]$ with noise, a modulus of wavelet coefficients $\left|d_{1}[n]\right|, \quad\left|d_{2}[n]\right|$, $\left|d_{3}[n]\right|, \quad\left|d_{4}[n]\right|, \quad\left|d_{5}[n]\right|$ and a smoothed signal $a_{5}[n]$ of five-level dyadic decomposition using digital filters from the Tables 3 and 4

Example 2. This example relates to the dyadic decomposition of the signals from Example 1 with the difference that the wavelet is the second derivative of a smoothing function (Fig. [10). The highpass filter associated with the wavelet is given in Table 7. Both the wavelet and the filter are symmetric at zero and do not introduce phase shift in signals $d_{k}[n]$. Singular points of the analyzed signal correspond to the places of intersection of signals $d_{k}[n]$ with the $\mathrm{x}$-axis (zero-crossings). Figures 19 and 20 illustrate the usefulness of dyadic multiscale analysis in detection 
of signal singularities, particularly in the case of noisy signals.

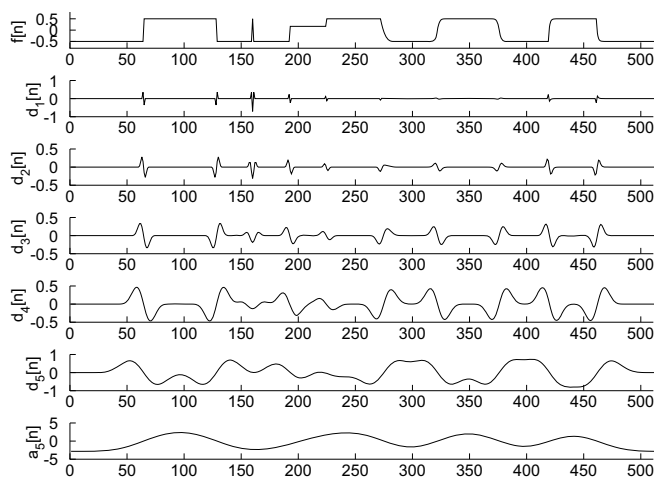

Fig. 19. Example of a digital signal $f[n]$, wavelet coefficients $d_{1}[n], d_{2}[n], d_{3}[n], d_{4}[n], d_{5}[n]$ and a smoothed signal $a_{5}[n]$ of a five-level dyadic decomposition using digital filters from Tables 3 and 7

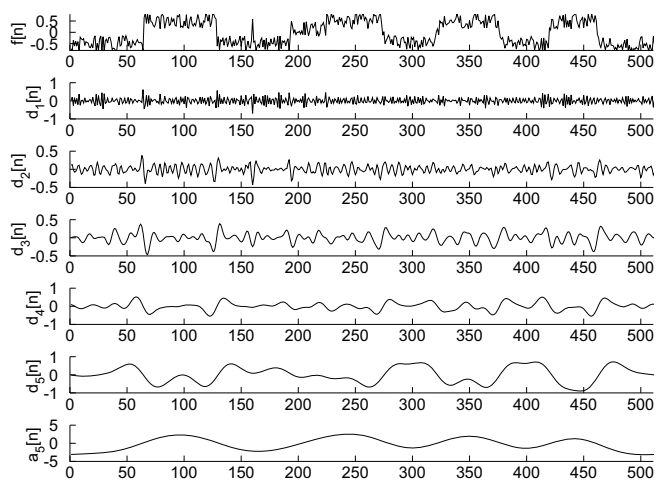

Fig. 20. Example of a digital signal $f[n]$ with noise, wavelet coefficients $d_{1}[n], d_{2}[n], d_{3}[n], d_{4}[n], d_{5}[n]$ and a smoothed signal $a_{5}[n]$ of five-level dyadic decomposition using digital filters from Tables 3 and 7

\section{Conclusion}

The paper presents a discrete dyadic wavelet decomposition of signals utilizing cubic box spline wavelets to detect the signal edges and allowing the signal singularity detection and calculation of a singularity measure in the form of Lipschitz exponent. Low-pass filter banks associated with cubic box spline wavelets used for wavelet decomposition do not shift the signal, thereby easing the tracking of lines that end at singular points of the signal. A cubic box spline wavelet that is the first derivative of the smoothing function introduces a phase shift that is easy to correct, and the cubic spline wavelet which is the second derivative of the smoothing function does not shift the signal at all.

Both the wavelets were applied for the dyadic decomposition of a noiseless as well as a noisy signal. The results of calculations illustrate the usefulness of cubic box spline wavelets for the detection of signal edges, particularly for noisy signals.

The singularity detection method presented in this paper utilises real wavelets with compact and short supports, and a fast algorithm for dyadic wavelet decomposition (algorithm à trous). Tu and Hwang (2005) show that complex-valued wavelets can be used to analyze signal singularities. Their paper constitutes an extension of Mallat's work on the subject discussed in this paper.

\section{Acknowledgment}

This work was supported by the Białystok University of Technology under the grant S/WZ/1/2014.

\section{References}

Babaud, J., Witkin, A.P. and Baudin, M. (1986). Uniqueness of the Gaussian kernel for scale-space filtering, IEEE Transactions on Pattern Analysis and Machine Intelligence 8(1): pp. 26-33.

Boor, C. (1978). A Practical Guide to Splines, Springer-Verlag, New York, NY.

Holschneider, M., Kronland-Martinet, R., Morlet, J. and Tchamitchian, P. (1989). A real-time algorithm for signal analysis with help of the wavelet transform, in J.-M. Combes, A. Grossmann and A.P. Tchamitchian (Eds.), Wavelets, Time-Frequency Methods and Phase Space, Springer-Verlag, Berlin/Heidelberg, pp. 286-297.

Mallat, S. (1991). Zero-crossings of a wavelet transform, IEEE Transactions on Information Theory 37(4): 1019-1033.

Mallat, S. (2009). A Wavelet Tour of Signal Processing: The Sparce Way, Third Edition, Academic Press, Burlington, MA.

Mallat, S. and Hwang, L.W. (1992). Singularity detection and processing with wavelets, IEEE Transactions on Information Theory 38(2): 617-643.

Mallat, S. and Zhong, S. (1992). Characterization of signals from multiscale edges, IEEE Transactions on Pattern Analysis and Machine Intelligence 14(7): 710-732.

Press, W.H., Teukolsky, S.A., Vetterling, W.T. and Flannery, B.P. (2007). Numerical Recipes The Art of Scientific Computing, Cambridge University Press, Cambridge.

Rakowski, W. (2003). A proof of the necessary condition for perfect reconstruction of signals using the two-channel wavelet filter bank, Bulletin of the Polish Academy of Science: Technical Sciences 51(1): 14-23.

Shensa, M.J. (1992). The discrete wavelet transform: Wedding the à trous and Mallat algorithms, IEEE Transactions on Signal Processing 40(10): 2464-2482. 
Skodras, A., Christopoulos, C. and Ebrahimi, T. (2001). The JPEG 2000 still image compression standard, IEEE Signal Processing Magazine 18(5): 36-58.

Tu, C.-L. and Hwang, W.-L. (2005). Analysis of singularities from modulus maxima of complex wavelets, IEEE Transactions on Information Theory 51(3): 1049-1062.

Unser, M. (1999). Splines: A perfect fit for signal and image processing, IEEE Signal Processing Magazine 16(6): 22-38.

Unser, M. and Blu, T. (2003). Mathematical properties of the JPEG2000 wavelet filters, IEEE Transactions on Image Processing 12(9): 1080-1090.

Witkin, A.P. (1983). Scale-space filtering, Proceedings of the International Conference on Artificial Intelligence, Karlsruhe, Germany, pp. 1019-1022.

Witkin, A.P. (1984). Scale-space filtering: A new approach to multi-scale description, in S. Ullman and W. Richards (Eds.), Image Understanding, Ablex, Norwood, NJ.

Zhao, Y., Hu, J., Zhang, L. and Liao, T. (2013). Mallat wavelet filter coefficient calculation, 5th International Conference on Computational and Information Sciences, Shiyan, Hubei, China, pp. 963-965.

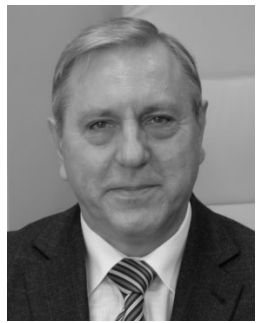

on computer graphics.
Waldemar Rakowski received the M.Sc. degree in computer science from the Faculty of Electronics, Warsaw University of Technology, in 1974, the Ph.D. degree from the Szczecin University of Technology in 1979, and the D.Sc. degree from the Łódz University of Technology in 2003. Since 2004, he has been a professor at the Białystok University of Technology. His research interests are focused on signal and image processing, in particular wavelets applications, and

Received: 27 June 2014

Revised: 3 April 2015 